\title{
Autopercepção de saúde: um estudo com idosos de baixa renda de São Paulo
}

\author{
Self-rated health: a study in an elderly low \\ income population of São Paulo
}

Thiago Rodrigo da Silva', Paulo Rossi Menezes²

\begin{abstract}
Silva TR, Menezes PR. Autopercepção de saúde: um estudo com idosos de baixa renda de São Paulo. Rev Med (São Paulo). 2007 jan.-mar.;86(1):28-38.

RESUMO: A autopercepção de saúde vem ganhando um importante espaço nas pesquisas epidemiológicas devido à sua forte e independente associação com mortalidade. Artigos internacionais apontam vários fatores determinantes da autopercepção de saúde, mas poucos estudos têm sido realizados em países em desenvolvimento, como o Brasil. Este trabalho tem como objetivo estudar os fatores socioeconômicos e as doenças que estão associadas à autopercepção de saúde. Foi realizado um estudo de corte transversal com 2.072 idosos acima de 65 anos, residentes em bairros da periferia oeste da cidade de São Paulo onde foram obtidos dados sobre características socioeconômicas, doenças crônicas, dificuldades nas atividades diárias, dor crônica e sintomas psiquiátricos menores, analisados com um modelo de regressão logística politômica. Foi encontrada associação da autopercepção de saúde com renda, angina, derrame, tosse crônica, dificuldades nas atividades diárias, dor e sintomas de transtornos mentais comuns. Concluímos que a autopercepção de saúde é uma variável muito consistente, e estudos realizados em países desenvolvidos encontraram resultados semelhantes.
\end{abstract}

DESCRITORES: Auto-imagem. Saúde do idoso. Envelhecimento. Doença crônica/ epidemiologia. Doença crônica/mortalidade. Fatores socioeconômicos.

\footnotetext{
* Trabalho premiado no COMU - Prêmio POC - Medicina Preventiva.

${ }^{1}$ Aluno do $4^{\circ}$ ano do curso de medicina da Faculdade de Medicina da USP.

2 Professor Associado do Departamento de Medicina Preventiva da Faculdade de Medicina da USP.

Endereço para correspondência: Rua Chico Herrera, 53 - Vila Nova Mazzei, São Paulo, SP, CEP: 02314-090. e-mail: throdrigo@uol.com.br
} 


\section{INTRODUÇÃO}

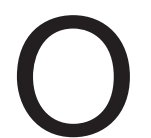
envelhecimento populacional é um fenômeno mundial, e o Brasil também tem observado essa mudança na sua pirâmide etária. Os países desenvolvidos já convivem com uma grande população de idosos há muitas décadas, mas os países em desenvolvimento, como - Brasil, têm assistido a esse fenômeno recentemente, fruto da queda nas taxas de natalidade e do aumento da expectativa de vida.

Dados do Instituto Brasileiro de Geografia e Estatística (IBGE) mostram que os idosos com 60 anos ou mais representavam $7,3 \%$ da população em 1991; e em 2000, representava $8,6 \%$ da população. Projeções do próprio IBGE mostram que em 2020 os idosos representarão $13 \%$ da população, excedendo o número de 30 milhões de pessoas, contra 14,5 milhões de idosos em $2000^{10}$.

Esse envelhecimento populacional certamente deverá refletir em uma mudança de estratégia em diversos setores do governo, principalmente no setor de saúde. Será necessário que o Brasil passe por uma importante reformulação tanto em gestão econômica ligada à saúde quanto em especialização e formação de profissionais capacitados a atender essa população, e produção de conhecimento científico que agregue uma melhor compreensão aos conhecimentos já estabelecidos, para que os anos vividos a mais sejam com a melhor qualidade possível'.

Nesse sentido, esse trabalho vem apresentar dados relevantes sobre autopercepção de saúde em idosos.

A autopercepção de saúde é uma variável não utilizada na clínica, mas muito utilizada em trabalhos científicos devido à sua forte associação com mortalidade, morbidade e uso de serviço médico. Ela tem mostrado associação com mortalidade independente de doenças crônicas referidas e fatores socioeconômicos, sendo essa associação é mais forte que qualquer outra variável objetiva.

Autopercepção de saúde (Self-Rated Health $\mathrm{SRH}$ ) é obtida questionando o indivíduo "como ele classifica a sua saúde nos últimos dias" e dando como opção de resposta cinco classificações, variando desde muito ruim, ruim, regular, boa e muito boa.

A autopercepção de saúde mantém-se constante durante a vida adulta até os 50 anos, quando entra em acelerada queda ${ }^{12}$, o que torna importante essa variável no cuidado com pacientes idosos, e faz com que a maioria dos estudos publicados foque essa população nos seus mais variados aspectos, muito embora existam trabalhos mostrando a importância da autopercepção de saúde em pacientes com câncer, por exemplo ${ }^{5}$.

Devido à importante associação entre autopercepção de saúde e mortalidade, a análise dos fatores determinantes da autopercepção de saúde se torna importante, pois modular esses fatores significa alterar a autopercepção de saúde e pode significar alterar a mortalidade. Os estudos publicados vêm mostrando que os principais fatores associados são os demográficos (idade, sexo, estado conjugal e arranjo familiar), os socioeconômicos (educação e renda), o número de doenças crônicas (hipertensão, artrite ou reumatismo, doença cardiovascular, diabetes, asma, bronquite ou enfisema, embolia ou acidente vascular cerebral e câncer), e a capacidade funcional (atividades de vida diárias) ${ }^{1}$. Ainda existe associação entre SRH e história familiar de doenças. Essa associação é controversa entre os estudos e esse aspecto controverso pode ser explicado por uma associação não direta com idade da morte dos pais ou causa da morte dos pais, mas sim associa SRH com o tipo de interação que a história familiar exerce com o padrão de cuidado com a saúde e o próprio estado de saúde que o indivíduo tem, em comparação com o padrão de cuidado à saúde e o estado de saúde que um familiar já falecido teve ${ }^{9}$. Todos esses fatores ligados à com autopercepção de saúde são claramente associados à morbidade e mortalidade, e juntos pesam na autoavaliação que o indivíduo faz sobre a sua saúde. Cabe aqui a reflexão sobre qual a razão de utilizar-se um índice que seja moldado pelos fatores que geram mortalidade e afirmar que esse índice é marcador de mortalidade? Ele não seria na realidade uma "variável de confusão" entre todos esses fatores associados e o índice de mortalidade. Existem dois argumentos que talvez possam falar a favor do uso da SRH e contra a tese de ser a autopercepção de saúde uma variável de confusão. O primeiro é que tanto a obtenção dos dados de todos esses fatores objetivos associados a $\mathrm{SRH}$, quanto a análise de todos eles, num sentido de observar qual a combinação entre eles que predispõe mais ou menos a uma alta mortalidade, é muito mais complexa que a simples obtenção da autopercepção de saúde e a quase imediata avaliação do risco de mortalidade que ela representa. Assim, a autopercepção de saúde funciona como uma representação, uma mensuração das avaliações objetivas de saúde ${ }^{2}$, sendo uma medida interessante para o uso na prática clinica. Instrumentos curtos de avaliação reduzem custos e são de interpretação mais fácil, e SHR é um exemplo de vantagem nesse sentido ${ }^{3}$. O segundo argumento a favor do SHR, é que os trabalhos publicados mostram que $\mathrm{SRH}$ tem um valor preditivo de mortalidade independente das avaliações objetivas 
de fatores de risco para mortalidade, como já citado anteriormente nesse texto. O mecanismo dessa associação permanece sem um esclarecimento, mas existem diversas hipóteses sendo levantadas, como por exemplo, inferência que a má autopercepção de saúde pode ser vinculada a um menor engajamento com práticas preventivas de saúde e menor adesão às recomendações médicas, mostrando um aspecto comportamental dessa associação. Outra hipótese é que a má autopercepção de saúde está associada à uma modulação negativa que o sistema nervoso pode fazer sobre o sistema imunológico, gerando suscetibilidade à doenças futuras ${ }^{9}$.

Ao fazer um levantamento de artigos, é possível notar que a maioria dos estudos publicada sobre o assunto foca populações residentes em países desenvolvidos, sendo bastante carente a investigação dessas informações em países subdesenvolvidos. Faz-se muito importante essa avaliação nesses países, pois existem diferenças socioeconômicas e diferenças de saúde e de doenças que poderiam dar um peso diferente à relação da autopercepção de saúde em e mortalidade encontrada em países desenvolvidos. Nos países subdesenvolvidos existe uma presença mais marcante de doenças infecciosas como causa de mortalidade, e mesmo outras causas agudas de mortalidade, que não guardam vínculo com a autopercepção de saúde do idoso. Nesse sentido, acredita-se que SRH teria um poder preditivo de mortalidade menor nos países subdesenvolvidos.

Somando-se a isso, nos países subdesenvolvidos a população tem menos informação sobre saúde em relação aos países desenvolvidos, tornando menos provável que o idoso faça uma boa avaliação de seu estado de saúde, e, possivelmente enfraquecendo a relação entre má autopercepção de saúde e mortalidade 6 .

Tendo em vista a importância da autopercepção de saúde e a falta de análise de populações de países subdesenvolvidos, investigamos dados de saúde, dados socioeconômicos, de dificuldades nas atividades diárias, da presença de dor crônica e de sintomas de transtornos mentais comuns em idosos de mais de 65 anos de uma região bastante carente do município de São Paulo.

\section{MATERIAIS E MÉTODOS}

\section{Desenho}

Foi feito um estudo observacional de corte transversal desenvolvido com base nos dados do projeto "Epidemiologia da demência e doença de Alzheimer em populações miscigenadas no Brasil e em Cuba".

\section{Amostra}

A amostra do estudo foi composta pela população com mais de 65 anos residente na região dos Distritos Administrativos do Butantã, Rio Pequeno e Raposo Tavares. A região tem 377.576 habitantes, dos quais $6,2 \%$ têm 65 anos ou mais, totalizando 23.410 idosos.

O tamanho da amostra foi calculado com base na associação esperada entre demência e miscigenação racial, devido ao objetivo principal do projeto "Epidemiologia da demência e doença de Alzheimer em populações miscigenadas no Brasil e em Cuba" e o resultado do cálculo apontou 2000 o número de indivíduos necessários.

\section{Instrumentos/medidas}

A autopercepção de saúde foi acessada com a pergunta: "De forma geral, como o sr/sra. classificaria a sua saúde nestes últimos 30 dias?" A resposta foi padronizada como "muito boa", "boa", "regular", "ruim" e "muito ruim".

As doenças crônicas avaliadas foram:

- hipertensão arterial, baseada na medida de pressão arterial com aparelho digital. A pressão foi medida três vezes, com o intervalo de 5 minutos entre as medidas. As medidas foram feitas com os entrevistados em abstinência de pelo menos uma hora de café e fumo, sentados e com o braço na altura do coração. A primeira medida foi descartada e foi calculada a média aritmética entre a segunda e terceira medidas. Foi considerado hipertenso pacientes com pressão diastólica maior ou igual a $90 \mathrm{mmHg}$ e/ou pressão sistólica maior ou igual a $140 \mathrm{mmHg}$ e/ou referência de diagnóstico anterior de hipertensão arterial em tratamento ou não;

- distúrbio cardíaco (infarto, angina, insuficiência cardíaca, valvopatia, outro), questionando o entrevistado se alguma vez foi informado por um médico que tinha problema cardíaco e qual foi o tipo de problema;

- acidente vascular cerebral, questionando o paciente se alguma vez teve um derrame que precisou de cuidados médicos;

- tosse crônica, através da história de tosse com catarro ao acordar nos meses de inverno, com duração mínima de três meses;

- diabete melito, definido pela presença de glicemia maior ou igual a $126 \mathrm{mg} / \mathrm{dL}$ e/ou pela referência de diagnóstico anterior de diabetes;

- claudicação intermitente, acessada pelo relato de dor nas panturrilhas ao andar, que faça o individuo andar devagar ou parar e desaparece em 10 minutos ou menos se ele parar. Foi usado o questionário para claudicação intermitente da OMS; 
- Angina, através da aplicação do "Rose angina questionnaire" da OMS, um instrumento de diagnostico de angina em trabalhos de campo, cuja sensibilidade e especificidade foram avaliadas em 33\% e $93,8 \%$, respectivamente.

A presença de dor crônica foi avaliada questionando o idoso com que freqüência no ultimo mês algum problema de saúde lhe causou dor: (1) nunca, (2) 2-3 vezes no mês passado, (3) mais ou menos uma vez por semana, (4) 2-3 dias por semana ou (5) todos os dias. Perguntamos também o grau de gravidade o idoso daria para a dor no ultimo mês: (1) leve, (2) moderada, (3) grave, (4) muito grave, (5) insuportável. E também perguntamos quando a dor está no pior momento, quanto ela impede de fazer as coisas: (1) nada, (2) não muito, (3) moderadamente, (4) muito. Foi calculado um score pela somatória dos pontos entre parênteses atribuídos a cada resposta, tendo o score valores entre 1 e 15.

A presença de sintomas psiquiátricos comuns foi avaliada pelo Self Report Questionnaire (SRQ-20), um questionário de 20 questões da Organização Mundial de Saúde que aponta como possível caso de transtorno psiquiátrico comum indivíduos do sexo masculino com seis ou mais respostas afirmativas e do sexo feminino com 7 ou mais respostas afirmativas. No Quadro 1, estão relacionadas as perguntas do teste.

Quadro 1. Self Report Questionnaire - SRQ-20

\begin{tabular}{|l|}
\hline \multicolumn{1}{|c|}{ SRQ } \\
\hline 1. Tem dores de cabeça freqüentes? \\
2. Tem falta de apetite? \\
3. Dorme mal? \\
4. Assusta-se com facilidade? \\
5. Tem tremores nas mãos? \\
6. Sente-se nervoso(a), tenso (a), ou preocupado(a)? \\
7. Tem má digestão? \\
8. Tem dificuldade de pensar com clareza? \\
9. Tem se sentido triste ultimamente? \\
10. Tem chorado mais do que de costume? \\
11. Encontra dificuldades para realizar com satisfação \\
suas atividades diárias? \\
12. Tem dificuldades para tomar decisões? \\
13. Tem dificuldades no serviço (seu trabalho é \\
14. Énoso)? \\
15. Temapaz de desempenhar um papel útil em sua \\
16. Você se sente uma pessoa inútil, sem préstimo? \\
17. Tem tido idéia de acabar com a vida? \\
18. Sente-se cansado(a) o tempo todo? \\
19. Tem sensações desagradáveis no estômago? \\
20. Você se cansa com facilidade? \\
\hline
\end{tabular}

Foi feita uma avaliação de desempenho do idoso nas atividades de vida diárias, questionando a presença e o grau da dificuldade de permanecer em pé por longos períodos, como 30 minutos, dificuldade com os afazeres domésticos, com aprender uma nova tarefa, em participar de atividades comunitárias, em se concentrar para fazer algumas coisas por 10 minutos, em andar longas distâncias, como um quilômetro, em lavar seu próprio corpo, em vestir-se, em lidar com pessoas desconhecidas, em manter amizade e dificuldade com o trabalho diário e as atividades usuais. Após essa avaliação, o idoso foi inquirido sobre o quanto essas dificuldades interferem na vida dele, obtendose a variável "interferência nas atividades de vida diárias"(IAVD). Foi feita uma análise preliminar e se percebeu a forte associação dessa última pergunta com as outras questões sobre cada dificuldade especifica, optando-se por trabalhar com a variável "interferência nas atividades de vida diárias" como uma forma de acessar o desempenho do idoso nas atividades de vida diárias.

\section{Procedimento}

Foi realizado um levantamento de campo procurando porta a porta domicílios com indivíduos de 65 anos ou mais. Foi necessário visitar em 21.727 domicílios, encontrando 2.266 idosos com 65 anos ou mais, dos quais 2072 aceitaram participar do estudo, com um índice de participação de 91,4\%. Todos os idosos encontrados na casa eram convidados a participar da pesquisa, e todos os idosos que aceitaram foram incluídos na pesquisa. Foi então agendada uma data especifica para cada idoso ser entrevistado. As entrevistas foram realizadas no domicílio do idoso (ou no local por ele indicado), e tiveram duração média de 2 horas e 30 minutos, respondendo o idoso um questionário amplo, com informações sociodemográficas, econômicas e de saúde geral, bem como foram submetidos a testes de declínio cognitivo, de capacidade funcional, de transtornos mentais comuns, exame físico geral e neurológico. Os entrevistadores foram treinados pelos coordenadores do projeto "Epidemiologia da demência e doença de Alzheimer em populações miscigenadas no Brasil e em Cuba" e utilizaram uma apostila de padronização da forma de conduzir as entrevistas e realizar os exames físicos. Durante a entrevista, agendava-se então uma nova data em que o idoso seria visitado por um auxiliar de enfermagem para coletar amostra de sangue para exames laboratoriais e genéticos. Esta fase de coleta de dados ocorreu no período de maio de 2003 a abril de 2005. 


\section{Análise}

Foram excluídos da análise 92 idosos cujas informações de autopercepção de saúde foram fornecidas por informantes, e um idoso que não soube responder à questão, utilizando-se então 1.979 indivíduos para análise, que representam $87,3 \%$ dos elegíveis.

A autopercepção de saúde foi classificada boa, composta pelas respostas de autopercepção "muito boa" e "boa"; em regular, composta pela resposta "regular" e ruim, composta pelas respostas "ruim" e "muito ruim". A variável idade foi dividida em quatro grupos, num intervalo de cinco em cinco anos, e a variável renda também em quatro grupos, dos quais três dividem a renda de $R \$ 0$ a $R \$ 700,00$, e um grupo contempla as rendas de $R \$ 701,00$ a $R \$ 7.000,00$, devido a baixa representatividade de indivíduos de alta renda. Da mesma forma, a educação foi dividida em três grupos, nos quais os idosos com quatro ou mais anos de educação formal estão num mesmo grupo, devido à baixa representatividade de indivíduos desse grupo.

A variável dor foi utilizada na análise utilizando as médias de scores de cada grupo analisado.

Foi utilizado o programa estatístico STATA para gerar tabelas de freqüência das variáveis e tabelas de associação entre as variáveis e a autopercepção de saúde, utilizando o teste de associação estatística do Qui quadrado $\left(\mathrm{X}^{2}\right)$. Foram geradas tabelas de risco relativo utilizando o método da regressão politômica. A partir da análise desses dados, foi criado o modelo de regressão logística multinomial com as variáveis associadas, e, foi criado um modelo de associação entre $\mathrm{SRH}$ e as variáveis de interesse.

\section{RESULTADOS}

Dos 1979 idosos no estudo, 60,69\% eram do sexo feminino, e a idade média foi de 71,92 , com $44,11 \%$ dos idosos incluídos na faixa de 65 a 69 anos de idade, e apenas $12,03 \%$ dos idosos na faixa etária superior a 80 anos. A renda encontrada foi bastante baixa, com $30,32 \%$ dos idosos ganhando menos de $\mathrm{R} \$ 240,00$, e apenas $25,42 \%$ dos idosos tendo uma renda maior que $\mathrm{R} \$ 701,00$. Os "anos de educação formal" encontrados nessa população foram igualmente baixos, com a maioria dos idosos (52,85\%) tendo estudado de 1 a 3 anos, e $37,49 \%$ não tiveram nenhum contato com educação formal.

Entre as doenças crônicas, a mais prevalente foi problema cardíaco, referido por $24 \%$ dos entrevistados. Durante a coleta dos dados, foi discriminado o tipo de problema cardíaco, e a insuficiência cardíaca respondeu por $28,2 \%$ dos problemas, enquanto na outra ponta, problemas valvares responderam por $0,08 \%$ dos problemas cardíacos. Hipertensão arterial foi encontrada em mais de $3 / 4$ da amostra (77,82\%), embora não foi possível obter a medida de pressão arterial e nem dado de uso de medicamento antihipertensivo em $2,07 \%$ dos entrevistados. A angina, diagnosticada pelo questionário da OMS, foi encontrada em 5,2\% dos idosos e o derrame em 8,39\%. Para o diagnóstico de diabetes, em $4,7 \%$ dos entrevistados não foi possível fazer medida de glicemia e nem obter resposta afirmativa de tratamento, ficando desconhecida a sua condição. No entanto, foi possível fazer diagnóstico de diabetes em 21,83\% dos entrevistados. Foi encontrada a prevalência de $5,36 \%$ de tosse crônica entre os idosos e $10,56 \%$ de claudicação intermitente.

A partir da Tabela 1, é possível observar associação entre autopercepção de saúde e quase todas as variáveis socioeconômicas e de saúde. Não foi encontrada associação somente entre autopercepção de saúde e idade, autopercepção de saúde e diabetes e autopercepção de saúde e claudicação intermitente.

Existe associação entre autopercepção de saúde ruim a variável "interferência nas atividades de vida diárias", onde quanto maior for a interferência que as dificuldades do idoso exercem na sua vida diária, maior é a chance de o idoso avaliar a saúde como ruim.

Pode-se perceber que existe associação entre autopercepção de saúde e SRQ. 22,53\% dos entrevistados preencheram critério como possível caso de transtorno mental comum. Nesse grupo, $23,54 \%$ dos indivíduos tiveram uma má autopercepção de saúde, enquanto no grupo em que não preenche critério para possível caso no $S R Q$, apenas $3,66 \%$ dos indivíduos relaram a saúde como ruim. A boa autopercepção de saúde foi maior entre os "não casos", com $54,97 \%$ de freqüência, contra $19,73 \%$ de freqüência de boa autopercepção de saúde entre os "casos". A autopercepção de saúde como regular sofreu uma menor variação entre os dois grupos, sendo ainda assim maior entre os casos do que entre os não casos. Não foi possível obter dados do SRQ de três pacientes, que se recusaram a terminar a entrevista ou não foi possível completála devido ao grau de distúrbio cognitivo do entrevistado.

O resultado do cruzamento das médias do Pain Score e a autopercepção de saúde, mostraram que associação, no sentido de quanto maior o Pain Score, maior a chance do idoso perceber a própria a saúde como ruim. 
Tabela 1. Freqüências de autopercepção de saúde vs características socioeconômicas, doenças crônicas, "interferência nas atividades diárias" (IAVD), pain, SRQ.

\begin{tabular}{|c|c|c|c|c|c|}
\hline \multirow[b]{3}{*}{$\mathbf{N}$} & \multirow{3}{*}{$\begin{array}{l}\text { Total } \\
1.979(100)\end{array}$} & \multicolumn{3}{|c|}{ Self-rated health } & \multirow[b]{2}{*}{$p$ value } \\
\hline & & Good & Fair & Poor & \\
\hline & & $930(47)$ & $888(44,9)$ & $161(8,1)$ & \\
\hline \multicolumn{6}{|l|}{ Socioeconomic characteristics } \\
\hline Gender & & & & & $<0,001$ \\
\hline Women & $778(39,3)$ & $512(42,6)$ & $573(47,7)$ & $116(9,7)$ & \\
\hline Men & $1.201(60,7)$ & $418(53,7)$ & $315(40,5)$ & $45(5,8)$ & \\
\hline Age (years) & & & & & 0,463 \\
\hline $65-69$ & $873(44,1)$ & $420(48,1)$ & $163(43,9)$ & $70(8)$ & \\
\hline $70-74$ & $542(27,4)$ & $261(48,1)$ & $232(42,8)$ & $49(9)$ & \\
\hline $75-79$ & $326(16,5)$ & $144(44,2)$ & $161(49,4)$ & $21(6,4)$ & \\
\hline 80 or more & $238(12)$ & $105(44)$ & $112(47)$ & $21(8,8)$ & \\
\hline Income $(R \$)$ & & & & & $<0,001$ \\
\hline $0-240$ & $600(30,32)$ & $222(37)$ & $308(51,33)$ & $70(11,67)$ & \\
\hline $241-360$ & $375(18,95)$ & $163(43,47)$ & $175(46,67)$ & $37(9,87)$ & \\
\hline $361-700$ & $501(25,32)$ & $255(50,9)$ & $211(42,12)$ & $35(6,99)$ & \\
\hline $701-7000$ & $503(25,42)$ & $290(57,65)$ & $194(38,57)$ & $19(3,78)$ & \\
\hline Education (Years) & & & & & $<0,001$ \\
\hline none & $742(37,5)$ & $324(43,7)$ & $352(47,4)$ & $66(8,9)$ & \\
\hline $1-3$ & $1046(52,8)$ & $488(46,6)$ & $475(45,4)$ & $83(7,9)$ & \\
\hline 4 or more & $191(9,6)$ & $118(61,8)$ & $61(31,9)$ & $12(6,3)$ & \\
\hline \multicolumn{6}{|l|}{ Chronic diseases } \\
\hline Hypertension & & & & & 0,016 \\
\hline No & $398(20,1)$ & $213(53,5)$ & $155(38,9)$ & $30(7,5)$ & \\
\hline Yes & $1540(77,8)$ & $700(45,4)$ & $710(46,1)$ & $130(8,4)$ & \\
\hline Unknow & $41(2,07)$ & - & - & - & \\
\hline Heart problem & & & & & $<0,001$ \\
\hline No & $1502(75,9)$ & $761(50,7)$ & $637(42,4)$ & $104(6,9)$ & \\
\hline Yes & $475(24)$ & $167(35,2)$ & $251(52,8)$ & $57(12)$ & \\
\hline Unknow & $2(0)$ & - & - & - & \\
\hline Angina (WHO Questionnaire) & & & & & $<0,001$ \\
\hline No & $1876(94,8)$ & $905(48,2)$ & $827(44)$ & $144(7,7)$ & \\
\hline Yes & $103(5,2)$ & $25(24,3)$ & $61(59,2)$ & $17(16,5)$ & \\
\hline Stroke & & & & & 0,008 \\
\hline No & $1813(91,6)$ & $849(46,8)$ & $826(45,6)$ & $138(7,6)$ & \\
\hline Yes & $166(8,4)$ & $81(48,8)$ & $62(37,3)$ & $23(13,9)$ & \\
\hline Diabetes & & & & & 0,44 \\
\hline No & $1454(73,5)$ & $697(47,9)$ & $640(44)$ & $117(8)$ & \\
\hline Yes & $432(21,8)$ & $193(44,7)$ & $205(47,4)$ & $34(7,9)$ & \\
\hline No data & $93(4,7)$ & - & - & - & \\
\hline Chronic cough & & & & & $<0,001$ \\
\hline No & $1872(94,6)$ & $900(48)$ & $832(44,4)$ & $140(7,5)$ & \\
\hline Yes & $106(5,4)$ & $29(27,4)$ & $56(52,8)$ & $21(19,8)$ & \\
\hline No data & $1(0)$ & - & - & - & \\
\hline Intermittent claudication (WHO questionnaire) & & & & & 0,239 \\
\hline No & $1770(89,4)$ & $840(47,5)$ & $783(44,2)$ & $147(8,3)$ & \\
\hline Yes & $209(10,6)$ & $90(43)$ & $105(50,2)$ & $14(6,7)$ & \\
\hline IAVD & & & & & $<0,001$ \\
\hline None & $1220(61,7)$ & $723(59,3)$ & $468(38,4)$ & $29(2,4)$ & \\
\hline Mildly & $231(11,7)$ & $69(29,9)$ & $138(59,7)$ & $24(10,4)$ & \\
\hline Moderately & $235(11,9)$ & $70(29,8)$ & $136(57,9)$ & $29(12,3)$ & \\
\hline Severely & $234(11,8)$ & $53(22,6)$ & $124(53)$ & $57(24,4)$ & \\
\hline Extremely & $58(2,9)$ & $15(25,9)$ & $21(36,2)$ & $22(37,9)$ & \\
\hline No data & $1(0)$ & - & - & - & \\
\hline Pain score & & & & & $<0,001$ \\
\hline Mean & 5,4 & 3,8 & 6,4 & 9,4 & \\
\hline Self Report Questionnaire (SRQ) & & & & & $<0,001$ \\
\hline No case & $1530(77,3)$ & $841(55)$ & $633(41,4)$ & $56(3,6)$ & \\
\hline Case & $446(22,5)$ & $88(19,7)$ & $253(56,4)$ & $105(23,5)$ & \\
\hline No data & $3(0,1)$ & - & - & - & \\
\hline
\end{tabular}


Foi criado um modelo de riscos relativos, o grupo de comparação foi onde o "desfecho" é a autopercepção de saúde boa. Comparou-se então o incremento (dado pelo risco relativo) na freqüência de um desfecho "adverso" (autopercepção da saúde como regular ou ruim) gerado por diferentes exposições à "fatores de risco" (características socioeconômicas, doenças crônicas, DAS, ADL's, $S R Q$, Pain Score), assim como se analisa o incremento (através do risco relativo) na incidência de AVC quando se compara tabagistas (expostos ao fator de risco) e não tabagistas (grupo de comparação). A diferença neste estudo, é que existem dois desfechos (autoavaliar a saúde como regular ou ruim) ao invés de apenas um desfecho (ocorrência de AVC), de modo que em cada tabela existam duas associações distintas (uma entre autopercepção de saúde ruim e boa, e outra entre autopercepção de saúde regular e boa). A Tabela 2 traz os resultados dessa análise univariada para as variáveis sociodemográficas e doença crônica. Foi encontrada uma redução do risco relativo $(\mathrm{RR})$ de autoavaliar a saúde como ruim nos homens em relação às mulheres de avaliar a saúde como boa. Essa redução é maior na chance de autoavaliar a saúde como ruim em relação a chance de autoavaliar a saúde como regular. A idade não esteve associada com a autopercepção de saúde, e a variável renda associou-se à autopercepção de saúde, e quanto maior a renda, menor é a chance de autoavaliar a saúde negativamente. No grupo onde a renda é de $R \$ 701,00$ a $R \$ 7.000,00$, houve uma proteção importante, com uma redução no risco relativo de $80 \%$ na chance de autoavaliar a saúde como ruim em relação ao grupo em que a renda é de $R \$ 0$ a $R \$$ 240,00 (grupo controle) avaliar a saúde como boa. Os anos de educação interagem com autopercepção de saúde, porém a diferença só é estatisticamente significante entre o grupo sem nenhum estudo formal e o grupo que estudou quatro ou mais anos, apresentando uma redução de $50 \%$ da chance do grupo mais estudado avaliar a saúde como regular e também $50 \%$ de redução de avaliar a saúde como ruim em comparação ao grupo sem estudo de avaliar a saúde como boa.

Tabela 2. Riscos relativos em análise multivariada.

\begin{tabular}{|c|c|c|c|c|c|}
\hline \multicolumn{6}{|c|}{ Self-rated health } \\
\hline & Good & Fair & $\mathrm{p}$ value & Poor & $p$ value \\
\hline \multicolumn{6}{|c|}{ Socioeconomic characteristics } \\
\hline Women & 1 & - & & - & \\
\hline Men & & 0,7 & $<0,001$ & 0,5 & $<0,001$ \\
\hline \multicolumn{6}{|l|}{ Income $(R \$)$} \\
\hline $0-240$ & 1 & - & & - & \\
\hline $241-360$ & & 0,8 & 0,067 & 0,7 & 0,149 \\
\hline $361-700$ & & 0,6 & $<0,001$ & 0,4 & $<0,001$ \\
\hline $701-7000$ & & 0,5 & $<0,001$ & 0,2 & $<0,001$ \\
\hline \multicolumn{6}{|c|}{ Education (Years) } \\
\hline none & 1 & - & & - & \\
\hline $1-3$ & & 0,9 & 0,274 & 0,8 & 0,316 \\
\hline 4 or more & & 0,5 & $<0,001$ & 0,5 & 0,036 \\
\hline \multicolumn{6}{|c|}{ Chronic diseases } \\
\hline \multicolumn{6}{|c|}{ Hypertension } \\
\hline No & 1 & - & & - & \\
\hline Yes & & 1,4 & 0,005 & 1,3 & 0,203 \\
\hline \multicolumn{6}{|l|}{ Heart problem } \\
\hline No & 1 & - & & - & \\
\hline Yes & & 1,5 & $<0,001$ & 1,9 & $<0,001$ \\
\hline \multicolumn{6}{|c|}{ Angina (WHO Questionnaire) } \\
\hline No & 1 & - & & - & \\
\hline Yes & & 2,7 & $<0,001$ & 4,3 & $<0,001$ \\
\hline \multicolumn{6}{|l|}{ Stroke } \\
\hline No & 1 & - & & - & \\
\hline Yes & & 0,8 & 0,172 & 1,7 & 0,028 \\
\hline \multicolumn{6}{|l|}{ Chronic cough } \\
\hline No & 1 & - & & - & \\
\hline Yes & & 2 & 0,002 & 4,7 & $<0,001$ \\
\hline \multicolumn{6}{|l|}{ IAVD } \\
\hline None & 1 & - & & - & \\
\hline Mildly & & 3,1 & $<0,001$ & 8,7 & $<0,001$ \\
\hline Moderately & & 3 & $<0,001$ & 10,3 & $<0,001$ \\
\hline Severely & & 3,6 & $<0,001$ & 26,8 & $<0,001$ \\
\hline Extremely & & 2,2 & 0,025 & 36,6 & $<0,001$ \\
\hline \multirow{2}{*}{\multicolumn{6}{|c|}{$\begin{array}{l}\text { No data } \\
\text { Self Report Questionnaire (SRQ) }\end{array}$}} \\
\hline & & & & & \\
\hline No case & 1 & - & & - & \\
\hline Case & & 3,8 & $<0,001$ & 17,9 & $<0,001$ \\
\hline
\end{tabular}


Quando se avaliam as doenças crônicas, percebe-se que com a variável hipertensão, ocorre um risco relativo de 1,4 do grupo hipertenso avaliar a saúde como regular. Não houve associação estatisticamente significativa no grupo hipertenso que avalia sua saúde como ruim. A variável tosse crônica teve uma forte associação no grupo com tosse crônica de autoavaliar a saúde como ruim, com um RR de 4,7.

A avaliação da variável "interferência nas atividades de vida diárias" (IAVD) mostrou que o existe associação com autopercepção de saúde regular em quem apresenta alguma interferência das dificuldades físicas nas atividades de vida, sendo que o valor da associação não apresentou um gradiente, ficando próximo do $R R$ de 3 . Já no grupo que classifica a saúde como ruim, pode-se perceber um gradiente de associação, onde quanto maior a interferência, maior o RR de avaliar a saúde como ruim, passando de um RR de 8,7 no grupo com pouca interferência e chegando em 36,6 no grupo com grande interferência.
Tendo o diagnóstico de provável caso de transtorno mental comum, o idoso tem um risco relativo de considerar a saúde como regular de 3,8 vezes maior que um não caso considerar a saúde como boa (IC 95\%: 2,9 - 5); e um risco relativo de considerar a saúde como ruim 17,9 vezes maior que um não caso considerar a saúde como boa (IC 95\%: $12,1-26,5)$.

A partir dos dados de risco relativo em análise univariada, foram criados modelos logísticos para avaliar a relação entre autopercepção de saúde (SRH) e cada uma das variáveis que se mostraram associadas, retirando o efeito confundidor das outras variáveis sobre a associação. A Tabela 3 traz os resultados da análise, e mostra que as variáveis sexo, idade, escolaridade, hipertensão e problema cardíaco perderam a associação na análise multivariada. Mantiveram-se associadas a renda, a angina, o acidente vascular cerebral (apenas no grupo com $\mathrm{SRH}$ regular), a tosse crônica (apenas no grupo com o SRH ruim), IAVD, dor e SRQ.

Tabela 3. Riscos relativos após análise multivariada por regressão logística multinomial.

\begin{tabular}{lllllll}
\hline & \multicolumn{5}{c}{ Self-rated health } \\
\cline { 2 - 7 } & \multicolumn{7}{c}{ Fair } & \multicolumn{3}{c}{ Poor } \\
\cline { 2 - 7 } & RR & p value & IC 95\% & RR & p value & IC 95\% \\
Income & 0,8 & $<0,001$ & $0,8-0,9$ & 0,7 & $<0,001$ & $0,6-0,9$ \\
Angina & 1,9 & 0,009 & $1,1-3,3$ & 2,5 & 0,014 & $1,2-5,3$ \\
Stroke & 0,5 & 0,001 & $0,4-0,8$ & 0,7 & 0,213 & $0,4-1,2$ \\
Chronic cough & 1,6 & 0,049 & $1-2,7$ & 2,5 & 0,012 & $1,2-5$ \\
IAVD & 1,2 & $<0,001$ & $1-1,3$ & 1,7 & $<0,001$ & $1,5-2$ \\
Pain score & 1,1 & $<0,001$ & $1,1-1,2$ & 1,3 & $<0,001$ & $1,2-1,3$ \\
SRQ & 2,4 & $<0,001$ & $1,8-3,2$ & 6,3 & $<0,001$ & $4-9,8$
\end{tabular}

\section{DISCUSSÃO}

A participação do estudo de $91,4 \%$ foi considerada adequada. Foram excluídos 92 indivíduos que não conseguiram responder sozinho - SRQ e a variável foi respondida por um informante. É provável que a dificuldade cognitiva desses indivíduos seja extrema. Walker et al. estudaram pacientes idosos com algum grau de distúrbio cognitivo, a fim de validar a relação entre autopercepção de saúde e mortalidade e mostrar qual - peso que a cognição pode ter nessa relação. Os autores encontraram que SRH é uma válida mensuração de mortalidade em pacientes com distúrbio cognitivo leve e moderado, no entanto, essa relação se perde em pacientes com distúrbio cognitivo grave, sendo a autopercepção de saúde uma variável menos interessante que avaliações objetivas de saúde nesse grupo de pacientes. A esse resultado foi atribuído ao fato de o idoso com um grau de distúrbio cognitivo muito intenso não tem condições de fazer uma autopercepção de saúde fidedigna, invalidando o efeito dela sobre a mortalidade. Com a exclusão dos 92 idosos, espera-se que essa associação não coerente entre o estado de saúde e a percepção que o idoso tem sobre ela não tenha acontecido com magnitude acentuada. Além disso, a autopercepção de saúde é uma análise subjetiva e pessoal, não sendo valida ao propósito desse estudo à percepção da saúde do idoso dada por um terceiro. Existem dados que apontam que em geral, um informante tende a avaliar a saúde do idoso pior do que o idoso avaliaria, gerando uma distorção na análise ${ }^{1}$.

No nosso estudo, optamos por dividir a autopercepção de saúde em três categorias, diferentemente da divisão clássica na literatura em dois grupos. Adotamos tal conduta, a fim de tentar observar de maneira mais refinada a associação entre as variáveis independentes e a autopercepção de 
saúde, tendo a capacidade de observar se existe um gradiente de associação.

Foi observado como tendência dos resultados existir uma associação maior das variáveis independentes com uma autopercepção de saúde ruim do que com uma autopercepção de saúde regular. $\mathrm{Na}$ tabela de freqüência, ao observar a variável "IAVD", é possível notar que os idosos que não possuem interferência das dificuldades na sua vida diária são distribuídos principalmente no grupo de autopercepção de saúde boa; os idosos que apresentam interferência moderada estão distribuídos principalmente no grupo que apresenta uma autopercepção de saúde regular, e os idosos que apresentam uma interferência extrema, estão distribuídos principalmente no grupo que apresenta uma autopercepção de saúde ruim. Futuros estudos que disponham de dados de mortalidade poderiam beneficiar-se dessa abordagem de subdivisão da autopercepção de saúde em três ou mais grupos, a fim de validar se esse gradiente permanece presente quando é analisado o óbito como desfecho de uma autopercepção de saúde boa, regular ou ruim.

Quando observamos a diferença entre os sexos, as mulheres avaliaram a saúde pior que os homens numa análise univariada. Esse resultado também foi encontrado por outros autores, que igualmente a esse estudo, observaram que quando se faz um modelo logístico, o sexo não está associado com a autopercepção de saúde. Embora o resultado por nos obtido corrobore com estudo de revisão sistemática sobre o tema, ainda não existe um consenso na literatura acerca da interação do gênero na autopercepção de saúde, já que existem trabalhos que encontram uma maior autopercepção de saúde ruim entre as mulheres ${ }^{12,16}$, e trabalhos que apontam uma maior associação entre má autopercepção de saúde e sexo masculino ${ }^{1}$, mesmo controlando a associação para outras variáveis possivelmente confundidoras.

No nosso estudo a idade não foi fator associado com autopercepção de saúde. Esse resultado já havia sido encontrado na literatura ${ }^{14}$, e contrariado por alguns estudos ${ }^{15}$, que demonstraram que a autopercepção de saúde era tanto pior quanto maior fosse a idade do individuo interrogado, e que esse efeito era percebido a partir dos 50 anos.

A renda do idoso apareceu no modelo como uma importante variável para a autopercepção da saúde. Isso ocorre provavelmente pela maior disponibilidade de recursos de saúde que os idosos que apresentam uma renda mais elevada possuem. Estudos estabelecem uma relação entre uma melhor autopercepção de saúde como um refletor da presença de recursos pessoais e sociais que possam atenuar o declínio da saúde ${ }^{9}$. Nesse sentido, renda é um importante recurso pessoal, bem como suporte social de vizinhos e amigos ${ }^{4,13}$. É possível que a autopercepção de saúde e renda contribua para o desaparecimento do modelo multivariado da variável "anos formais de educação". Na realidade, mais de quatro anos de educação estaria associada com uma boa autopercepção de saúde pelo vínculo que os anos formais de educação têm com a renda. Alves e Rodrigues ${ }^{1}$ encontram a escolaridade como fator determinante da autopercepção de saúde, diferente do que encontramos em nossa amostra.

Classicamente, os estudos avaliam a associação entre autopercepção de saúde e doenças crônicas comparando indivíduos com nenhuma doença crônica e indivíduos com certa quantidade de doença(s) crônica(s). Essa metodologia não nos permite analisar a influência de cada doença na autopercepção de saúde. Nesse sentido, incorporamos à literatura dados interessantes que apontam quais doenças estão associadas com autopercepção de saúde e quais doenças não estão associadas.

Desde o início do estudo a diabetes não se mostrou associada à autopercepção de saúde, bem como a claudicação intermitente. Já o idoso que tinha problema cardíaco relatava uma autopercepção de saúde pior que o idoso sem problema cardíaco, mas essa diferença perdeu-se com a análise multivariada. O mesmo efeito ocorreu com a hipertensão arterial, que durante o estudo mostrou um comportamento interessante. Observou-se a análise univariada uma associação somente entre autopercepção de saúde regular e hipertensão, e a análise multivariada não encontrou associação entre hipertensão e autopercepção de saúde. Dados colhidos no estudo e não publicados nesse trabalho, nos revelaram que é possível encontrar associação entre autopercepção de saúde e hipertensão arterial sistêmica somente num subgrupo de hipertensos em que a hipertensão arterial interfere muito na vida do individuo, Como a maioria dos pacientes possuem hipertensão arterial não complicada, que é por si é um distúrbio essencialmente assintomático, é possível que essa associação encontrada num subgrupo específico de hipertensos seja diluída pelo efeito da não associação entre hipertensão leve e autopercepção de saúde, que é mais representativa na amostra.

As doenças que permaneceram associadas à autopercepção de saúde (angina, AVC e tosse crônica) foram doenças que de alguma forma limitam ou interferem na vida do idoso. Considerar esse como um possível critério de associação com autopercepção de saúde pode ser validado quando se observa a hipertensão arterial, que é assintomática não esta associada com a autopercepção de saúde, mas logo perde a validade quando se observam as outras doenças que não foram associadas com 
autopercepção de saúde e que também geram limitação ou interferência na vida do idoso, como é o caso da claudicação intermitente. Assim, não foi possível, com os nossos dados, explicar os resultados obtidos com as doenças crônicas, sendo importante que a literatura futura adote a metodologia de analisar a relação individual de cada doença com a autopercepção de saúde, a fim de validar ou contrariar os nossos resultados quando outras amostras populacionais forem abordadas e quais os fatores que influenciam nesses resultados.

A variável dor manteve-se associada com a autopercepção de saúde após a análise multivariada. Esse resultado era esperado, pois Mantyselka et al. ${ }^{14}$ demonstraram em 2003 uma forte associação entre autopercepção de saúde e dor crônica, independente de doenças crônicas, sexo e idade.

O SRQ está fortemente associado com a autopercepção de saúde, indicando que transtornos mentais comuns apresentados pelo idoso são determinantes da autopercepção de saúde. Esse resultado tem sido reproduzido na literatura ${ }^{7,11}$, inclusive demonstrando que os transtornos mentais modulam intensamente a autopercepção de saúde mesmo quando eles estão somente nos antecedentes pessoais do idoso e não são manifestos no momento em que se afere a sua autopercepção de saúde ${ }^{11}$.

A interferência que as dificuldades do idoso (permanecer em pé por longos períodos, como 30 minutos; dificuldade com os afazeres domésticos, com aprender uma nova tarefa, em participar de atividades comunitárias, em se concentrar para fazer algumas coisas por 10 minutos, em andar longas distâncias, como um quilômetro; em lavar seu próprio corpo, em vestir-se, em lidar com pessoas desconhecidas, em manter amizade e dificuldade com o trabalho diário e as atividades usuais) gera na sua vida, indicada pela variável "IAVD" estiveram associadas com a autopercepção de saúde. A literatura científica aponta que uma queda na capacidade funcional do idoso associa-se com uma percepção ruim de saúde ${ }^{1,17} \mathrm{em}$ todos os trabalhos que avaliam esse dado. Além disso, Walker et al. demonstraram que o declínio cognitivo no idoso está associado com a autopercepção de saúde, no entanto ele só é valido como mensuração de mortalidade em idosos com declínio leve ou moderado. Em idosos cujo declínio cognitivo é severo, a autopercepção de saúde perde força de associação com mortalidade, pois esses idosos não estavam em condições de fazer um bom julgamento da própria condição de saúde.

Finalmente, concluímos que muitos dos fatores associados à autopercepção de saúde na literatura internacional também são aplicados à uma população de baixa renda da cidade de São Paulo. Isso tem uma importância grande, pois pudemos observar a consistência da variável $\mathrm{SRH}$, que se mantém associada a uma boa parte dos fatores que indicam evolução de saúde ruim nos idosos e essa associação transcende aspectos culturais, econômicos e sociais. Cabe aos pesquisadores nacionais conhecerem essa variável tão poderosa e investigarem novas associações, bem como encontrar pistas que possam explicar os mecanismos que formam essas associações. É tarefa também a reprodução dos dados internacionais de forte preditor de mortalidade que a autopercepção de saúde possui, a fim de validar esse achado em nossa população.

AGRADECIMENTOS: Agradecemos aos coordenadores e pesquisadores do projeto "Epidemiologia da demência e doença de Alzheimer em populações miscigenadas no Brasil e em Cuba” e à estatística Letícia M. S. Coutinho pela grande contribuição na execução das análises estatísticas dos dados.

Silva TR, Menezes PR. Self-rated health: a study in an elderly low income population of São Paulo. Rev Med (São Paulo). 2007 jan.-mar.;86(1):28-38.

\begin{abstract}
Self-rated Health has been taking an important status in epidemiological research because of its strong and independent association with mortality. International papers indicate many determinants factors of self-rated health, but few studies have been carried out in developing countries as Brazil. This paper aims to study socioeconomic characteristics and chronic diseases associated with self-rated health. It was performed a cross-sectional study with 2,072 old aged adults over 65 years, living in an economically deprived area in São Paulo city. Socioeconomic characteristics, chronic diseases, difficulty with activities of daily living, chronic pain and common mental disorders symptoms were accessed and analyzed with a polytomic logistic regression model. We found an association of self-rated health and income, angina, stroke, chronic cough, difficulty with activities of daily living, chronic pain and common mental disorders symptoms. It was concluded that self-rated health is a consistent variable, and studies performed in developed countries have found similar results.
\end{abstract}

KEY WORDS: Self concept. Health of the Elderly. Aging. Chronic disease/epidemiology. Chronic disease/mortality. Socioeconomic factors. 


\section{REFERÊNCIAS}

1. Alves LS, Rodrigues RN. Determinantes da autopercepção de saúde entre idosos do Município de São Paulo, Brasil. Rev Panam Salud Publica. 2005;17:333-41.

2. Apples A, Bosma H, Grabauskas V, Gostautas A, Sturmans F. Self-rated health and mortality in Lithuanian and Dutch population. Soc Sci Med. 1996;42:681-9.

3. Bowling A. Just one question: if one question works, why ask several? J Epidemiol Community Health. 2005;59:342-45.

4. Cummins S, Stafford M, Macintyre S, Marmot M, Ellaway A. Neighbourhood environment and its association with self rated health: evidence from Scotland and England. J Epidemiol Community Health. 2005;59:207-13.

5. Fayers PM, Sprangers MA. Understanding self-rated health. Lancet. 2002;19:187-8

6. Frankenberg E, Jones NR. Self-rated health and mortality: Does the relationship extend to a low income setting? J Health Soc Behav. 2004;45:441-52.

7. Han, B. Depressive symptoms and self-rated health in community-dwelling older adults: a longitudinal study. J Am Geriatr Soc. 2002;50:1549-56.

8. Hansen MS, Fink P, Frydenberg M, Oxhoj ML, Sondergaard L, Eriksen M. Mental disorders in Medical inpatients and the association to severity of illness, selfrated physical disability, and health perception. Psychosomatics. 2001;42:41-7.

9. Idler EL, Benyamini Y. Self-Rated health and mortality: a review of twenty-seven community studies. J Health Soc Behav. 1997;38:21-37.

10. Instituto Brasileiro de Geografia e Estatística. Disponível em: http://www.ibge.gov.br.

11. John U, Meyer C, Rumpf HJ, Schumann A, Dilling H, Hapke U. Self-rated general health and psychiatric disorders in a general population sample. Eur Psychiatry. 2005;20:223-8.

12. Kubzansky LD, Berkman LF, Glass TA, Seeman TE. Is educational attainment associated with shared determinants of health in the elderly? Findings from the MacArthurt strudies of successful aging. Psychosom Med. 1998;60:578-85.

13. Lund R, Avlund K, Modvig J, Due P, Holstein BE. Development in self-rated health among older people as determinant of social relations. Scand J Public Health. 2004;32:419-25.

14. Mantyselka PT, Turumen JHO, Ahonen RS, Kumpusalo EA. Chronic pain and poor self-rated health. JAMA. 2003:290:2435-42.

15. McCullough ME, Laurenceau JP. Gender and natural history of self-rated health: a 59-year longitudinal study. Health Psychol. 2004;23:651-55

16. McDonough P, Walters V. Gender and health: reassessing patterns and explanations. Soc Sci Med. 1993;36:33-46.

17. Walker JB, Maxwell CJ, Hogan DB, Ebly EM. Does selfrated health predict surival in older persons with cognitive impairment? J Am Geriatr Soc. 2004;52:18951900. 